\title{
A training system for Industry 4.0 operators in complex assemblies based on virtual reality and process mining
}

\author{
Juan Jesús Roldán*, Elena Crespo, Andrés Martín-Barrio, Elena Peña-Tapia, Antonio Barrientos \\ Centre for Automation and Robotics (UPM-CSIC), Technical University of Madrid, José Gutiérrez Abascal, 2, Madrid 28006, Spain
}

A B S T R A C T

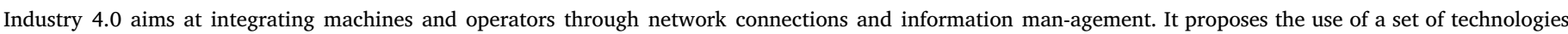

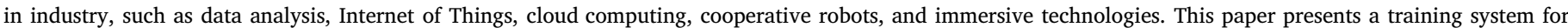

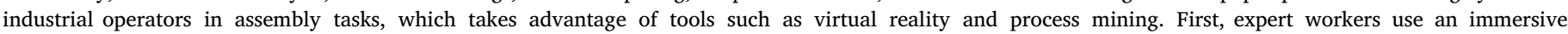

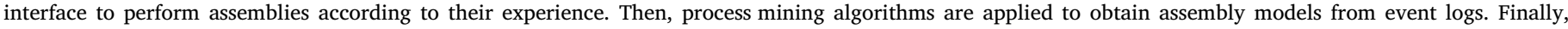

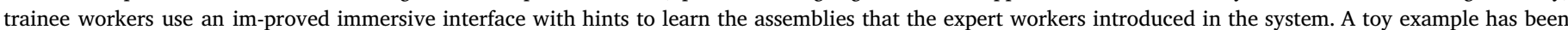

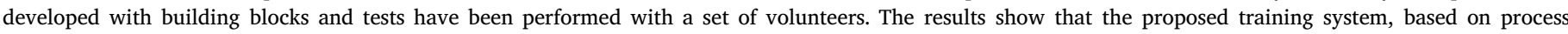

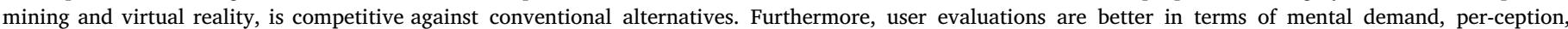
learning, results, and performance.

Keywords:

Industry 4.0

Training system

Assemblies

Virtual reality

Process mining

\section{Introduction}

Industry 4.0 can be considered as a paradigm shift in the industry that aims at combining all the production agents (machines, robots, and operators) in the shape of Cyber Physical Systems by means of network connections and information management [1]. Nine technologies are considered in the core of Industry 4.0 [2]: the analysis of large amounts of data, application of autonomous and cooperative robots, extensive simulation of processes, horizontal and vertical integration of systems, industrial Internet of Things, cloud storage and computing, additive manufacturing, virtual and augmented reality, and cybersecurity.

One of the active challenges of the industry is the management of knowledge [3]. Expert operators acquire valuable knowledge about the manufacturing processes over the years. For instance, this knowledge can be related to the efficient management of processes or the dis assembly, inspection, maintenance and assembly of machines. The transference of this knowledge to new operators is a key issue. How ever, this transference is sometimes not efficient or even does not take place, endangering the future of organizations [4].

This work aims at developing a system to facilitate this knowledge transference in the context of complex assembly tasks. For this purpose, it considers two of the main Industry 4.0 technologies: data analysis and virtual reality. In the first case, process mining techniques are applied to model the procedures followed by experts, store this knowledge and allow the apprentices to learn them. In the second case, virtual reality is used to provide experts and apprentices with an immersive environ ment to respectively convey their knowledge and learn the tasks.

The main developments of the work are listed below:

- Expert mode: It allows expert operators to perform assemblies in an immersive environment with natural interactions, capturing their sequences of actions in event logs.

- Data processing: Process mining algorithms are used to discover the assembly models from the data collected by event logs.

- Training mode: It provides new operators with an immersive en vironment with intuitive hints to learn the procedures that must be followed in the assemblies.

A set of experiments were performed to validate the proposed system. In these experiments, a series of volunteers had to study four assemblies: two of them using the immersive system and the other two by means of a physical instruction set. Then, they had to perform the assemblies as fast as possible and without mistakes. The results show that operators have at least the same performance using the proposed system than the conventional one and, moreover, they evaluate the experience more positively.

\footnotetext{
Conflict of interest: None

* Corresponding author.

E-mail address: jj.roldan@upm.es (J.J. Roldán).
} 
As far as we know, this is the first proposal of a training system for industrial operators that uses process mining and virtual reality. Process mining allows to collect and manage the knowledge of assembly pro cesses, whereas virtual reality provides expert and trainee operators with immersive and intuitive interfaces. The idea of the work is to develop an integral and automated solution for knowledge transfer in industry, taking advantage of both technologies to overcome the cur rent challenges of the industry. This system reduces the costs of working in the real environment, which can lead to temporally stop the pro duction or damage the equipment.

The remainder of the paper is organized as follows: Section $2 \mathrm{col}$ lects the main state of the art related to the use of virtual reality and process mining for training systems. Section 3 describes the developed system with its three modules: expert mode, data processing, and training mode. Section 4 reports the experiments performed to validate the system using simple assemblies. Section 5 discusses the results of these experiments and their implications. Finally, Section 6 summarizes the main conclusions of this study and its potential future works.

\section{State of the art}

Industry 4.0 provides new possibilities for assembly systems [5], such as aided assembly, intelligent storage management, self config ured workstation layout, product and process traceability, late custo mization and assembly control systems. Aided assembly is the closest among these concepts to this work, involving the assistance to workers during assemblies. For this purpose, technologies related to the Internet of Things are applied, such as placing sensors in every workstation, tool and product, and providing communications among all of them. Some examples of this operator support are the use of lights to show which object to pick or where to place it, and the application of virtual and augmented reality.

As mentioned above, two resources related to Industry 4.0 are used in this work: virtual reality to recreate the scenario and allow the work of operators (see Section 2.1), and process mining to discover the models behind their actions (see Section 2.2).

\subsection{Virtual reality}

Nowadays, three main immersive technologies can be distinguished: virtual reality (VR), augmented reality (AR) and mixed reality (MR) [6]. VR generates virtual scenarios where the users can interact with virtual elements. AR works in real scenarios, augmenting certain information by means of virtual elements and allowing the interaction with these elements. MR is a mixture of VR and AR that combines virtual and real worlds and allows virtual and real interactions.

A previous paper demonstrates that adequate immersion can im prove the perception and knowledge of operators [7]. In that paper, a VR interface is compared against a conventional one in the context of multi robot missions. The results show that the VR interface sig nificantly improves the situational awareness of operators without in creasing their workload. Additionally, the research supports the po tential of VR systems in the collaborative design and presentation of objects [8], the virtual manufacturing and testing of products [9], and the development and validation of complex products [10].

The literature contains several references about training systems based on virtual reality. One of the first studies [11] compares VR against conventional systems in the context of laparoscopic surgery. The results show that the students trained with VR are faster and made fewer mistakes than the ones trained with conventional procedures. More recent medical research works confirm these results in the fields of surgery [12] and imagery [13]. Additionally, some works propose VR based tools to analyze human factors in the context of manu facturing, such as ergonomic issues [14] and performance character istics [15].

A survey of stress assessment in military missions by using VR environments can be found in [16]. Other work proposes a virtual shooting training system for soldiers and reports that students have more motivation and better scores using it [17]. However, the level of immersion of this system is limited because the authors use a projector instead of a head mounted display, which reduces the field of view. A system to train visual scanning tasks is developed in [18], where sol diers have to find people around them and distinguish between civilian and enemies. The results show the importance of a realistic recreation to reduce the gap between simulation and reality and get better results. Finally, a social system to train veterans with post traumatic stress to face job interviews is proposed in [19]. This system is evaluated posi tively by the users, who report a significant rise in self confidence during the process.

In the context of industry, VR and AR technologies are compared for training industrial operators in maintenance and assembly tasks [20]. Four systems are analyzed: VR, VR with an explanatory video, AR and AR with a similar video. The results are favorable to AR against VR, but it must be remarked that the VR system uses a screen instead of a more immersive device. Other works use virtual reality to train operators to follow safety procedures in the mining industry [21], pointing out the importance of a wider field of view for a better performance [22]. Augmented reality is used to inspect the planned trajectories for ma nipulator robots, checking if they are safe and efficient before their execution [23]. However, virtual reality is also used to support the operators and control the robots in the assembly and manipulation of micro devices [24]. Additionally, the study reported by [25] directly compares a screen with a head mounted device, concluding that the second device is more immersive, intuitive, easy to use, interactive and easy to learn. Finally, [26] proposes a VR environment to train opera tors for welding processes, whereas [27] uses a similar environment in the context of a mechanized industry. In both cases, VR environments are recreated using screens instead of more immersive devices, but the experiments show the usefulness of these environments and the re levance of providing operators with adequate feedback of their actions.

\subsection{Process mining}

Process mining is a branch of data analysis that encompasses the modeling, evaluation and enhancement of processes from the events generated by them and collected in logs [28].

Therefore, there are three main concepts in process mining: pro cesses, event logs, and models. Processes are the object of study and generate huge amounts of data. Event logs collect the activities per formed by resources. Models represent processes and can be generated through events. Similarly, there are three types of techniques of process mining: discovery of models from event logs, conformance checking and model enhancement, and reproduction of models to generate event logs.

Process mining works with multiple types of models, such as tran sition systems [29], Petri nets [30], Business Process Modeling Notation [31] and causal nets [32].

Some use cases of process mining can be found in the following references: health care processes [33], public services [34], manu facturing processes [35] and robot missions [36]. The authors of these publications apply process mining techniques to analyze and optimize diverse processes. The knowledge is collected by event logs, which register every activity performed by every resource during the pro cesses. Then, they apply discovery algorithms to automatically model processes from the event logs. These models are used to analyze the resource allocation and time performance for the different processes. In this way, bottlenecks can be solved and processes can be optimized.

However, as far as we know, process mining has not been used to collect the knowledge of expert operators and transfer it to new workers in the context of industry. As the cited works, this one starts from a process (the assemblies performed by experts operators) and has to generate a model (the guide that trainee operators must follow). The 


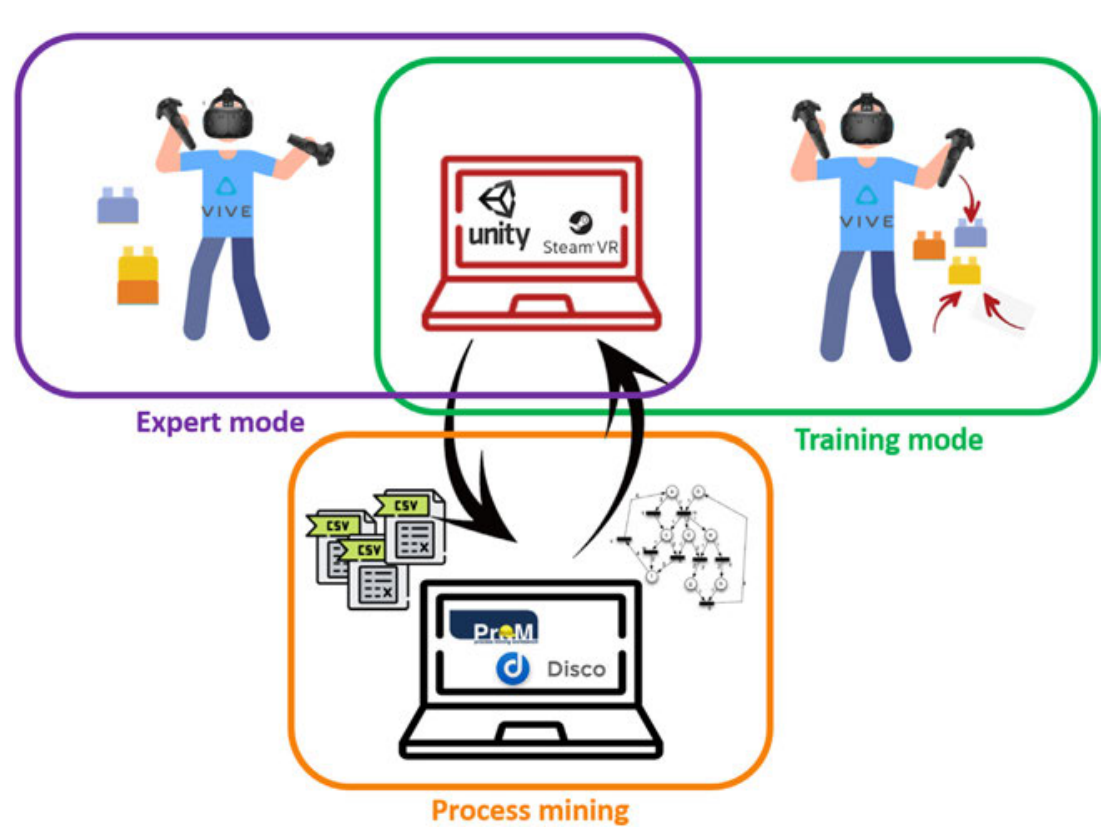

Fig. 1. System diagram: expert mode allows the expert operators to introduce their knowledge in the system, process mining automatically generates the assembly models from the operation logs, and training mode allows the new operators to learn the assembly.

main difference is that knowledge is not a means to optimize the pro cess, but an objective to be transmitted between operators.

\section{Description of system}

As already pointed out, the proposed system allows the transference of knowledge of assembly tasks from expert operators to new ones in the context of industry.

Fig. 1 shows an overview of the proposed system, which works in the following way. First, expert operators freely perform assemblies within the virtual reality scenario. The movements, decisions, and ac tivities of these operators are registered by the system and stored in event logs. Then, process mining is used to automatically generate transition systems from these event logs. The resulting models represent the different assemblies as sequences of states and transitions. Next, trainee operators are introduced in the virtual reality scenario to learn the assemblies. Using assembly models, the interface provides them with valid sequences of activities, showing which blocks to take and where to place them.

In this section and the following ones, the assemblies are considered to be performed by using the building blocks shown in Fig. 2. This assumption simplifies the development, description, and validation of the training system, but it does not limit the application of this system

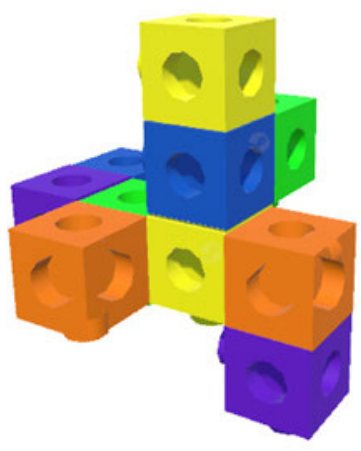

(a)

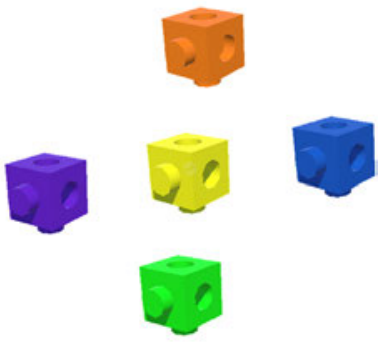

(b)
Fig. 2. One of the assemblies (a) and the blocks used to build it (b). to more complex and realistic assemblies. In fact, the system can be applied to assemblies that consist of blocks with different shapes, sizes and number of joints. The scope of this work includes the development of a prototype of the training system and its evaluation within a con trolled environment. From this perspective, this toy example is enough to reach preliminary conclusions. In future works, the system will be improved with information obtained from the experiments and tested with a real industrial case.

The remainder of this section is organized as follows: Section 3.1 describes the expert mode, Section 3.2 the application of process mining to model the assemblies and Section 3.3 the training mode.

\subsection{Expert mode}

This mode is designed to collect the knowledge of expert workers. In general, these expert workers are operators that have a certain ex perience in the assembly processes. This experience can be measured by the time spent working in a certain task and the number of assemblies performed. The specific values will depend on variables related to the assemblies, such as their complexity, length, and required precision. In principle, in order to save the knowledge of the process, an operator performing an assembly once is enough. However, the higher number of expert operators taking part in this process, the more quality and fewer deviations the stored information will present.

As already mentioned, this mode starts with some experts per forming repeated times the same assembly according to their experi ence. They do not have to build the assemblies always in the same way. In fact, performing different activities to arrive at the same state leads to a better fit of models (i.e., them representing reality more accu rately). Additionally, it provides the apprentice workers with the pos sibility to choose the best sequence of actions according to their pre ferences.

A coding system is necessary to unambiguously represent every possible activity and state of assemblies. Note that an activity means taking a block or set of blocks and join it to another block or set of blocks, whereas a state is the result of performing an activity. This paper presents an original strategy for coding the assemblies whose main rules are explained below:

- The blocks are identified by capital letters that depend on their 


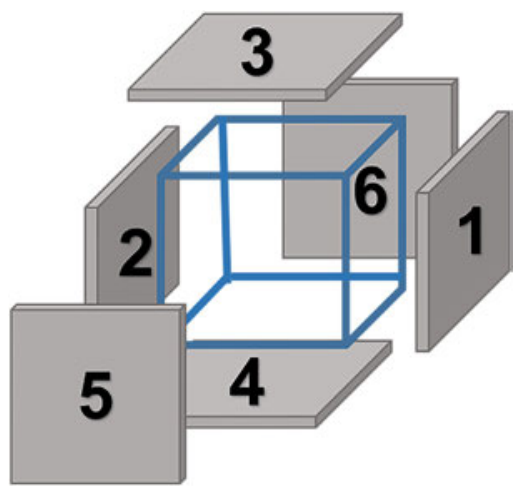

Fig. 3. Coding system for the faces of blocks.

types. For instance, we can assign names to the blocks of Fig. 2 (b) in the following way: A to green, B to blue, C to purple, D to orange and $\mathrm{E}$ to yellow ones. It is assumed that in an assembly like the one shown in Fig. 2 (a), the blocks of the same type are interchangeable.

- All the faces of the cube shaped blocks are considered as joints and numbered, in order to adequately represent the joints between them. Fig. 3 shows this notation applied to a cube that is similar to the blocks considered in the work: right is 1 , left is 2 , up is 3 , down is 4 , front is 5 and back is 6 . This notation is generalizable to any block independently of its shape, size, and number of faces.

- The assembly that results through joining two blocks is named by using the names of the blocks and their contact faces. For instance, when A is assembled down B, the notation of assembly is A34B.

- The name of an assembly must not depend on the sequence of ac tivities performed to build it. Following the previous example, when B is assembled over A (instead of A down B), the notation of as sembly must be again A34B. This can be addressed by using the following criteria to order the states after the activities:

- The main chain of the code is the longest.

- If two chains have the same length, the one lower index faces is chosen first. For example, a chain will be chosen as principal if it has more connections of type 12 than 21 . In the case of a tie, higher index faces are evaluated: 34 vs 43 and 56 vs 65 .

- The branches of the main chain are named by order of occurrence.

- The secondary branches are named with their correspondent primary branch and before passing to the following primary branch.

Fig. 4 shows some examples of application of the proposed rules. The first one shows how to obtain the main chain and order the bran ches. The second one is a special case where there are two equal blocks in the main chain (two Bs) and the one that has the branch must be specified ([2]B means the second B in the direction of the main chain). The third one shows the priority of 12 connections over 21 ones and the fourth the priority of 1221 criterion over 5665 one. The fifth one is a complex case where there is a tie in 1221 connections and the decision is based on 5665 criterion. Finally, the sixth example shows a case with all the possible connections (12 21, 3443 and 56 65) and the correct name of the assembly.

Back to the explanation of expert mode, it provides the expert workers with an immersive environment to build the assemblies. As shown in Fig. 5, the scenario reproduces a room with a table, where the blocks are initially placed, and a blackboard, which shows information about the assembly code and time. However, if the system is applied to real industrial cases, the environment can be adapted to faithfully re present real facilities.

The environment has been designed with Unity game engine and SteamVR plugin to be reproduced with an HTC Vive head mounted display. The scenario has been modeled by using the Unity application and some open source assets, whereas the blocks have been designed using a computer aided design application and then imported to Unity. The behavior of the different elements in the scenario is managed by some scripts developed in C\# using Unity and SteamVR libraries. The HTC Vive headset includes a head mounted display, which allows the user to get images and sounds of a virtual environment, two base sta tions, which are used to get the position and orientation of the head mounted display, and two hand controllers, which allow the users to configure the virtual environment and interact with their objects. The SteamVR plugin is necessary to allow the integration of the developed code with the head mounted display and hand controllers. All the data generated by the expert operators is stored locally in a set of spread sheets.

As previously described, the operators can freely do the assemblies in this mode and the system just registers their activities. When a worker takes a block, moves it to another one and joins both blocks, the system generates an event and stores it in an event log. As usual in process mining, the events are described by the following fields:

- Case ID: It is a unique identifier for each case, which allows dis tinguishing not only the different assemblies performed by the dif ferent resources, but also the different executions of the same as sembly performed by the same resource.

- Timestamp: It indicates the instant when the event is completed, for instance, using the format hh:mm:ss.

- Activity: It represents the type of event that takes place in the as sembly. A number is assigned to each event that appears starting from 1 and increasing it. If the same activity is executed twice, it only receives a number the first time.

- State: Similarly, it is a number that represents the result of the performed event. Again, if two different sequences of activities generate the same state, this state receives its corresponding number the first time.

An example of event log can be seen in Table 1. All the activities and states are new in the first case, whereas some of them are repeated in the second case. In this example, there are two ways to perform the assembly represented by cases 1 and 2, which follow the sequences of activities 12345 and 12367 and finish at the same state 5 .

Although these event logs are valid to represent processes and generate models, the training system needs to know which activities and states correspond to each number. For this purpose, other two logs are created: activity and state logs.

An example of activity log is shown in Table 2 and their fields are explained below:

- Activity ID: It is the unique identifier of the activity, which corre sponds to the one that appeared in the event log.

- Active assembly: It is the block or set of blocks that is taken by the user to start the activity.

- Target assembly: It is the block or set of blocks where the user joins the active assembly.

- Approach: It represents the type of joint between the two assemblies by using their contact faces.

- Active block: When the assembly chosen by the user has two or more blocks, it specifies the contact block in the active assembly.

- Target block: In the same case, it defines the contact block in the target assembly.

In the same way, an example of state log is shown in Table 3 and their fields are explained below:

- State ID: It is the unique identifier of the state, which corresponds to the one appeared in the event log.

- State code: It represents all the assemblies with two or more blocks that are on the table at the given moment separated by dashes. 


\section{Example 1:}

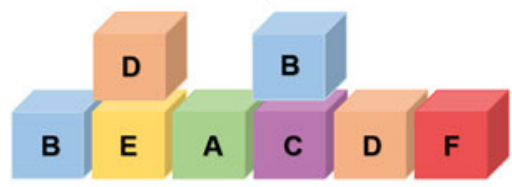

B12E12A12C12D12F/E34D/C34B

Example 3:

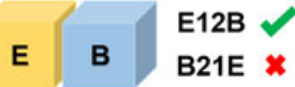

Example 5:

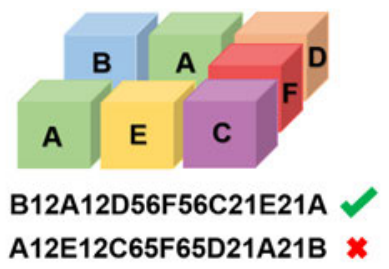

Example 2:

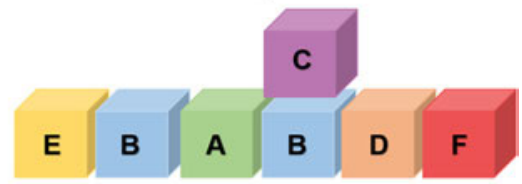

E12B12A12B12D12F/[2]B34C

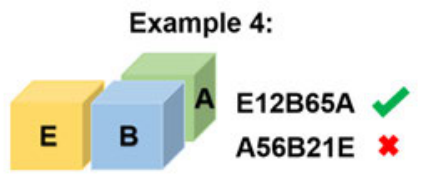

Fig. 4. Examples of assemblies and their names according to the coding system.

A video of the expert mode can be seen at this link.$^{1}$

\subsection{Data processing}

Once the operations of expert workers have been collected in event logs, it is necessary to obtain models from them. Although event logs include the sequences of activities and states, they may present in frequent behaviors that can be caused by errors or inefficiencies of operators. Additionally, event logs only contain relationships of pre cedence among the events, whereas the models are able to describe the causal and parallel relationships among them. For instance, if an event $\log$ represents 100 paths of 1234 and 50 paths of 132 4, a model can infer that 2 and 3 are parallel states, since they can be reached in a different order. Nevertheless, if an event log represents 150 paths of 1 234 and 1 path of 1324 , a model can discover that 2 is the cause of 3 and discard the anomalous path.

The first step is filtering the log to remove infrequent behaviors, which can be found in a frequency study like the shown in Fig. 6 (a). Although these behaviors may represent correct operations in the as semblies, they can also reveal operator inefficiencies and errors. Ad ditionally, the logs can be studied from a time perspective, for instance, finding the activities that spend more time, which can be seen in Fig. 6 (b). These activities sometimes reveal process bottlenecks that should be adequately managed. In this work, we used Disco to apply these filters to the data. This tool allows to define limits to the number of states and transitions, as well as thresholds in frequency and time to consider or discard events.

The second step is to generate models through the filtered event logs. In order to do this, a discovery algorithm of process mining must be used. In previous work, the Inductive Miner algorithm implemented in ProM was found the best algorithm in the context of robot missions [37]. This algorithm was able to generate models from all the con sidered logs, reaching a remarkable fitness together with a good bal ance between generalization and precision. For this reason, this algo rithm has been used in the present work, obtaining good models for all the cases. It must be remarked that the algorithm uses event logs to generate transition systems, whereas the activity and state logs are used in the following steps to interpret these models and give advice to op erators.

An example of transition system obtained through one of the event logs is shown in Fig. 7. As it can be seen, the transition system is similar to the event log (compared with Fig. 6). This fact is due to the lack of parallel activities, so all the precedence relationships in the event log are represented by causal ones in the transition system. The transition system is required by the training mode to properly guide trainee op erators.

\subsection{Training mode}

This mode is developed to train new workers to build complex as semblies. The interface is similar to the previously used in the expert mode: it uses the same room, table, and blackboard. However, this mode does not allow the operators to freely build the assemblies. Conversely, untrained operators can only perform activities that are coherent with the previously obtained models.

In order to do this, the transition systems are converted to task logs and read by the interface. An example of task log is shown in Table 4 and their fields are explained below:

- Initial state: It indicates the state of the assembly before the action of the operator.

- Activity: It represents the action of the operator.

- Final state: It indicates the state of the assembly that is reached after the action of the operator.

Given a certain state of the assembly, the operator can continue taking one or multiple blocks. These blocks are highlighted by the in terface by remarking their outlines in white, as shown by Fig. 8 (a). Once the operator takes one of them, there can be one or more possible destinations. In this case, the interface remarks the contact faces of active and target blocks with red arrows, as it can be seen in Fig. 8 (b). Note that our toy example considers blocks with fixed orientations, and this information was given to operators before the experiments.

A video of the training mode can be seen at this link. ${ }^{2}$ 


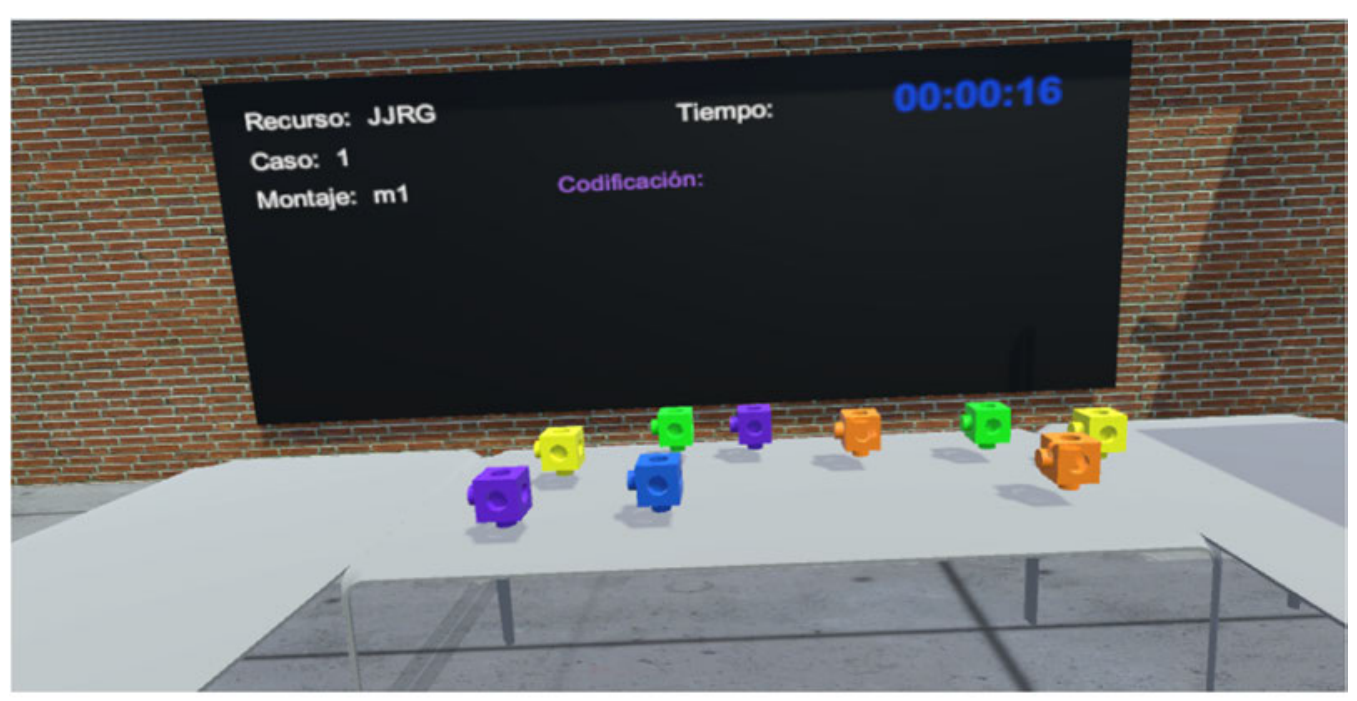

(a)

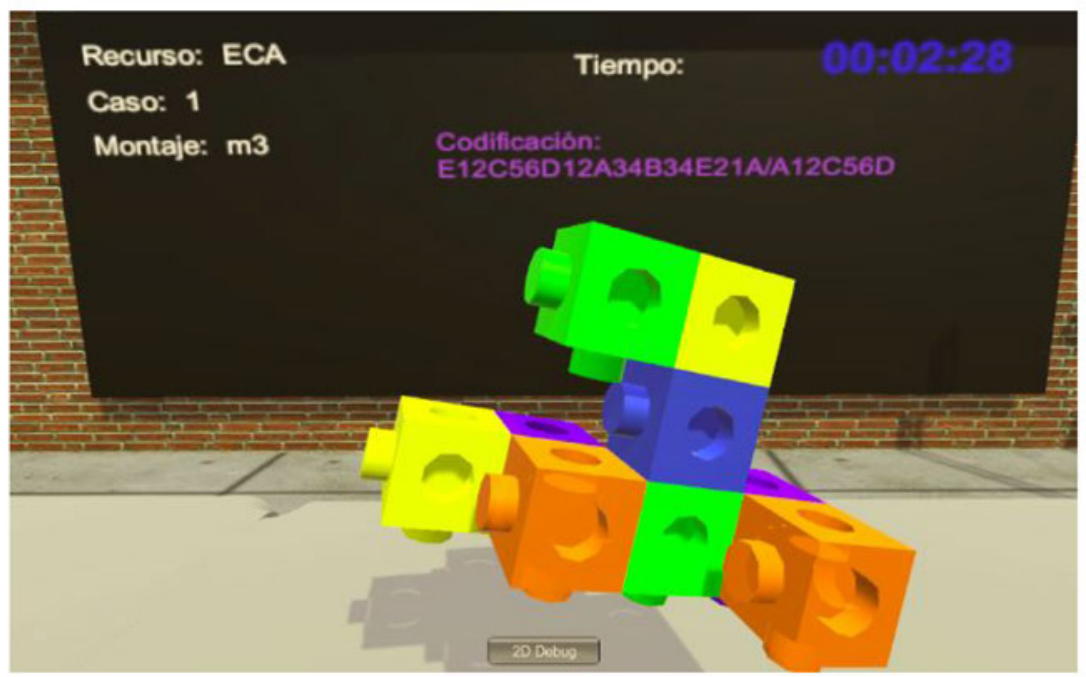

(b)

Fig. 5. Two views of scenario: (a) starting the assembly, (b) finishing it.

Table 1

Event log of an assembly.

\begin{tabular}{llll}
\hline Case ID & Timestamp & Activity & State \\
\hline 1 & $0: 00: 10$ & 1 & 1 \\
1 & $0: 00: 15$ & 2 & 2 \\
1 & $0: 00: 21$ & 3 & 3 \\
1 & $0: 00: 31$ & 4 & 4 \\
1 & $0: 00: 36$ & 5 & 5 \\
2 & $0: 00: 11$ & 1 & 1 \\
2 & $0: 00: 16$ & 2 & 2 \\
2 & $0: 00: 22$ & 3 & 3 \\
2 & $0: 00: 31$ & 6 & 6 \\
2 & $0: 00: 47$ & 7 & 5 \\
$\cdots$ & $\cdots$ & $\cdots$ & $\cdots$ \\
\hline
\end{tabular}

\section{Experiments}

A set of experiments was performed to validate the training system and compare its performance against a conventional method based on abstract instructions. These experiments were aimed at answering the following question: "Does the proposed training system provide better performance results and operator evaluations than a conventional
Table 2

Activity $\log$ of an assembly.

\begin{tabular}{llllll}
\hline ID & Act. assembly & Tar. assembly & Approach & Ac. block & Tar. block \\
\hline 1 & D & C & 56 & D & C \\
2 & E & A & 43 & E & A \\
3 & C & B & 21 & C & B \\
4 & D56C & A34E & 12 & C & E \\
5 & B12C & D56C12E43A & 65 & B & A \\
6 & B12C & A34E & 65 & B & A \\
7 & E43A56B12C & D56C & 21 & E & C \\
$\cdots$ & $\cdots$ & $\cdots$ & $\cdots$ & $\cdots$ & $\cdots$ \\
\hline
\end{tabular}

one?". For this purpose, we designed the four assemblies of Fig. 9, taking into account the difficulty levels, number of blocks and assembly paths collected in Table 5. We introduced these assemblies in the system by using the expert mode and repeating each of them at least 10 times following different instructions.

Twenty volunteers took part in the experiments: thirteen men and seven women with ages between 21 and 48. The volunteers were a diverse set of members from our university: ten BSc students, five MSc students, four $\mathrm{PhD}$ candidates and one professor. Taking into account 
Table 3

State $\log$ of an assembly.

\begin{tabular}{ll}
\hline State ID & State code \\
\hline 1 & D56C \\
2 & A34E-D56C \\
3 & A34E-B12C-D56C \\
4 & B12C-D56C12E43A \\
5 & D56C12E43A56B12C \\
6 & D56C-E43A56B12C \\
$\cdots$ & $\cdots$ \\
\hline
\end{tabular}

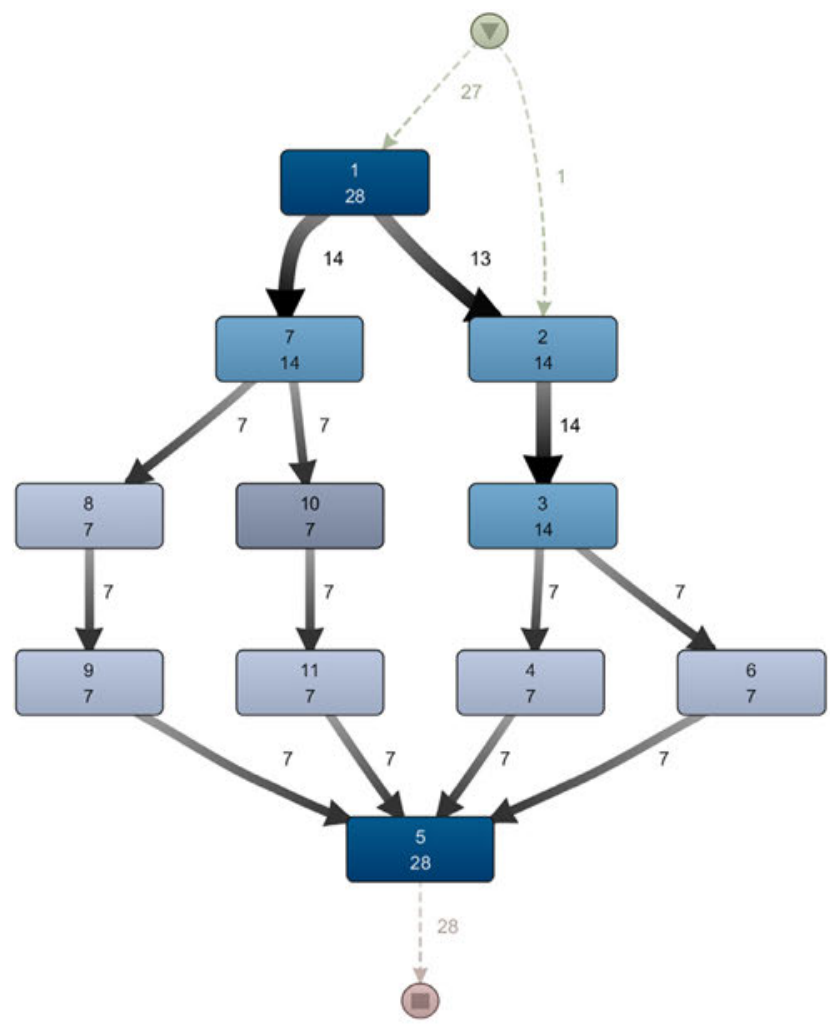

(a) that equation, the penalties assigned to these errors were $20,15,5$ and 10 seconds respectively.

Score $=T+5^{*} E_{O}+10^{*} E_{H}+15^{*} E_{L}+20^{*} E_{P}$

On the other hand, the subjective experience of every operator was evaluated by means of a questionnaire. This questionnaire had the following structure:

- Personal data: Name, age, gender, and studies.

- Previous experience: Questions about the experience of volunteers

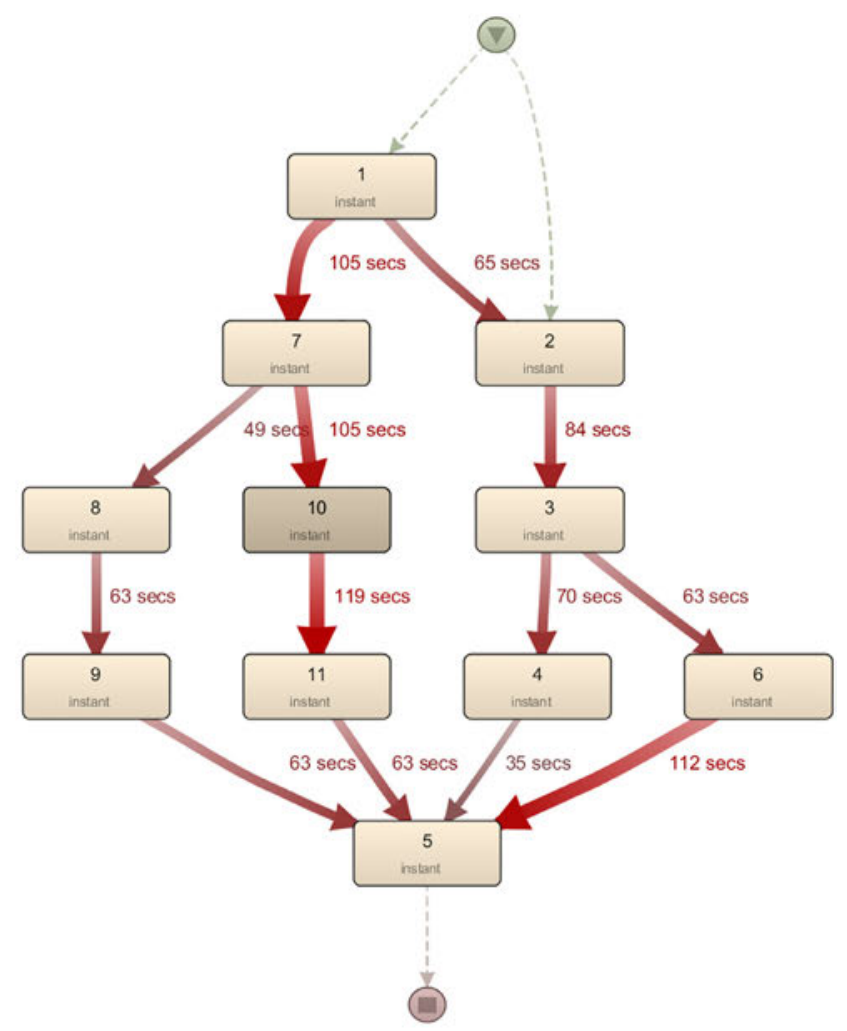

(b)

Fig. 6. Flowcharts of event log: (a) frequency analysis, (b) time analysis.

similar experiments performed in previous works, such as [7], we considered twenty operators are enough to perform a first analysis and reach relevant conclusions. Each of these participants had to do the four assemblies mentioned above, studying two of them by using the im mersive training system and the other two with a conventional guide, both of them shown in Fig. 10. We gave them 3 minutes to study the assemblies 1 and 2, considered easier, and 6 minutes to learn the as semblies 3 and 4, evaluated as harder. Both the assemblies and guides were ordered in the tests seeking to eliminate the influence of learning on results. After they learned an assembly by using the conventional or immersive guide, they had to build it with 3D printed blocks, as shown in Fig. 10 (c).

Two evaluations were performed in the experiments: one related to operator performance and another that involved operator experience.

On the one hand, as shown in Eq. (1), the performance of each operator was computed with the time spent to perform the assembly $(T)$ and the number of errors during it. Four types of errors were con sidered: the operator takes a wrong block $\left(E_{P}\right)$, the operator places a block in a wrong location $\left(E_{L}\right)$, the operator changes the order of two activities $\left(E_{O}\right)$ and the operator demands a hint $\left(E_{H}\right)$. As also shown in with building blocks and virtual reality.

- Evaluation of systems: Selection of the best training system in terms of mental demand, physical demand, temporal demand, effort, performance, frustration, perception, learning, and result. The first six variables are taken from NASA TLX workload questionnaire [38], which is widely used in robot missions and other contexts. The last three variables evaluate three important aspects that are not considered by that questionnaire: the spatial perception of assem blies, learning facility, and subjective evaluation of results. The training systems are ranked instead of rated because it is better to obtain significant results, according to the work in [39]. The vari ables can take five values: 1 means "VR guide is much better", 2 "VR guide is a little better", 3 "both guides are similar", 4 "conventional guide is a little better" and 5 "conventional guide is much better".

- Experiments: A set of questions about the time given to study the assemblies and the design of both training systems.

- Observations: A field to write free comments and suggestions. 


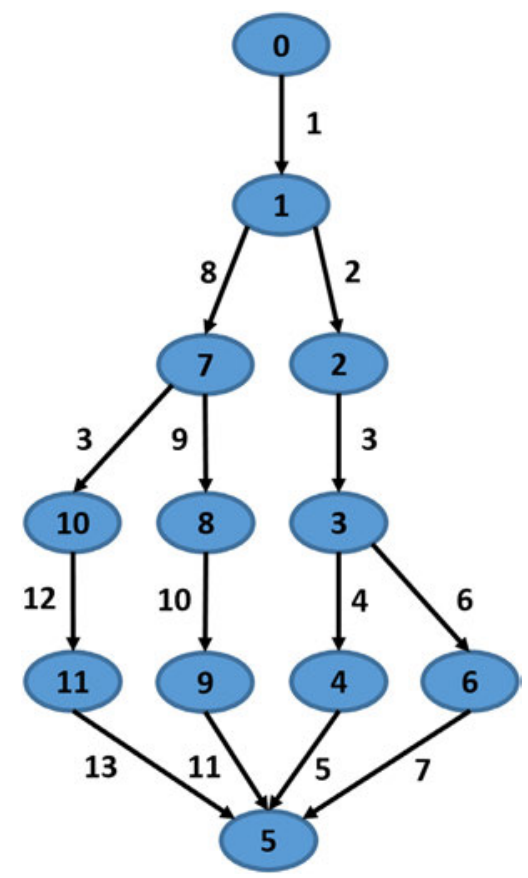

Fig. 7. Transition system obtained from the previous event log.

Table 4

Task $\log$ of an assembly.

\begin{tabular}{lll}
\hline Initial state & Activity & Final state \\
\hline 0 & 1 & 1 \\
1 & 8 & 7 \\
1 & 2 & 2 \\
7 & 3 & 10 \\
7 & 9 & 8 \\
2 & 3 & 3 \\
10 & 12 & 11 \\
8 & 10 & 9 \\
3 & 4 & 4 \\
3 & 6 & 6 \\
11 & 13 & 5 \\
9 & 11 & 5 \\
4 & 5 & 5 \\
6 & 7 & 5 \\
\hline
\end{tabular}

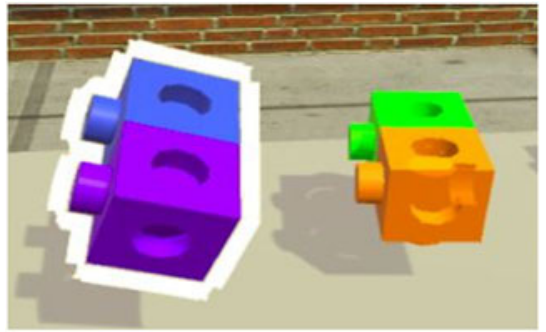

(a) discussion about the viability of the proposed training system in other scenarios is performed in Section 5.3.

\subsection{Performance analysis}

The performance scores of the trials are collected in Table 6. As it can be seen in the means and standard deviations of scores, the varia bility caused by operators is high and this can endanger the significance of results. In order to prevent this phenomenon, the results have been normalized applying Eq. (2). Thus, each normalized score represents the performance of an operator $o$ in an assembly $a$ divided by the sum of his/her scores in all the assemblies $i=\{1,2,3,4\}$. In this way, this metric represents the real cost for operators of each combination of assembly and guide related to all the performed assemblies. The nor malized performance scores are also contained in Table 6 .

NormScore $_{o, a}=\frac{\text { Score }_{o, a}}{\sum_{i=1}^{4} \text { Score }_{o, i}}$

Given the guide and assembly have influence on the results, the analysis must be performed taking into account both factors. As shown in Table 6 and Fig. 11, the assemblies can be ordered according to the obtained scores in the following way: $1,3,2$ and 4 . This is an un expected result because the assemblies were designed considering an increasing difficulty level: $1,2,3$ and 4 . Nevertheless, the operators probably found more difficulties to memorize and build the second assembly than the third one. A set of one way analyses of variance (ANOVA) was performed in order to check the significance of these results, revealing assembly 1 has less score than $2(p=0.0161)$ and 4 $(p=0.0000)$, assembly 2 has less score than $4(p=0.0005)$, and as sembly 3 has less score than $4(p=0.0000)$. No significant differences with $\alpha=0.05$ can be found between assemblies 1 and $3(p=0.0679)$, as well as between assemblies 2 and $3(p=0.3157)$.

As also shown in Table 6 and Fig. 11, the immersive training system exhibit better results than the conventional one in the easier assemblies ( 1 and 2 ), but the opposite occurs in the harder ones ( 3 and 4 ). The one way ANOVA reveals the difference is significant in assembly 1 ( $p=0.0295$ ), but not relevant in the rest of assemblies ( $p$ values of $0.1554,0.5392$ and 0.5770 for assemblies 2, 3 and 4 respectively). Therefore, it can be asserted that the proposed immersive training system is competitive against the conventional one.

These results do not match with our previous beliefs. VR systems are usually considered to provide a better spatial perception in complex

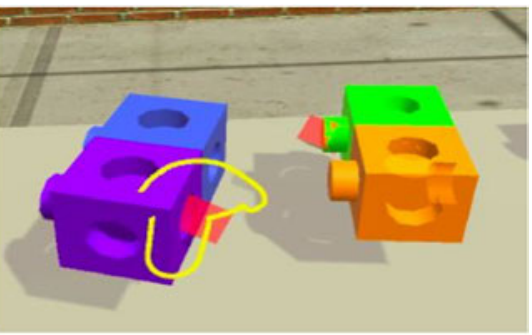

(b)

Fig. 8. Hints in training mode: (a) which block to take and (b) where to place it.

\section{Results}

As previously pointed out, twenty volunteers were asked to learn four assemblies, two with an immersive training system and other two with a conventional instruction set, and then build them using the 3D printed blocks, taking note of their times and errors. Then, the volun teers were asked to evaluate the training systems by answering a questionnaire. The performance analysis is addressed in Section 5.1, whereas the operator evaluations are analyzed in Section 5.2. Finally, a environments. However, the performance has been higher in the sim pler assemblies. We think this fact can be due to the experiment design. Although the assemblies were small, the volunteers found difficulties to distinguish between the blocks, since they only differ in colors. This fact caused the required times for training and building were short and the potential of the VR system was not exploited.

Finally, if volunteers that have previous experience with virtual reality (13) and those that are not familiar with this technology (7) are separated, it is possible to find an interesting result: the first ones have 


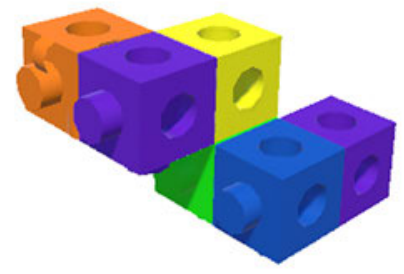

Assembly 1

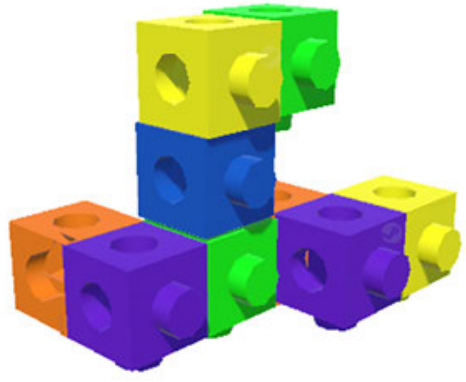

Assembly 3

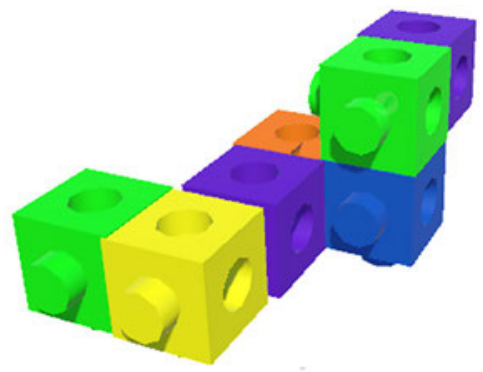

Assembly 2

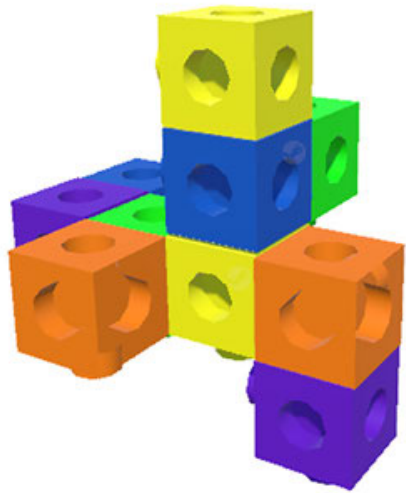

Assembly 4

Fig. 9. The four assemblies considered for the experiments ordered by their complexity (number of blocks).

Table 5

The four assemblies with their features.

\begin{tabular}{lllll}
\hline Assembly & Difficulty & Blocks & Expert paths & Trainee paths \\
\hline 1 & Low & 6 & 4 & 4 \\
2 & Low-Medium & 7 & 3 & 9 \\
3 & Medium-High & 9 & 2 & 4 \\
4 & High & 10 & 1 & 1
\end{tabular}

better performance with the VR guide (a mean of 0.2162 with a stan dard deviation of 0.1471 ) than with the physical one (a mean of 0.2838 with a standard deviation of 0.1286 ), whereas the second ones reach better results with the physical guide (mean 0.2238 and standard de viation 0.1490 ) than with the VR guide (mean 0.2762 and standard deviation 0.1446 ). The difference of the volunteers that have previous experience with VR is significant with alpha $=0.10$. This result supports the proposed immersive training system, since it indicates that the performance of operators using this system can be improved with adequate training.

\subsection{Operator evaluation}

The evaluations of operators are collected in Table 7 and shown in Fig. 12. As explained above, these evaluations are comparative: 1 means "VR guide is much better", 2 "VR guide is a little better", 3 "both guides are similar", 4 "conventional guide is a little better" and 5 "conventional guide is much better". At first glance, the results reveal that the VR guide is considered better than the conventional one in all the variables except physical demand, which can be explained with requiring standing and moving throughout the room.

The one way ANOVA of these results reveals the immersive training system is significantly better than the conventional one in terms of mental demand $(p=0.0036)$, performance $(p=0.0492)$, perception $(p=0.0000)$, learning $(p=0.0005)$ and result $(p=0.0241)$. The rest of results cannot be considered as relevant assuming $\alpha=0.05$.

As can be easily checked, the evaluations of operators are more favorable to the immersive training system than their performance scores. Every experiment has a subjective factor and, in this case, it has influence on the results and can provide interesting conclusions. This subjective evaluation of the training system, which does not depend on the objective results, can ease its future application.

Finally, the suggestions and claims from the users were collected during the experiments. Some of them suggested to include undo, redo and restart options in both interfaces. As a result, we will add these functionalities in the next version of the training system.

\subsection{Discussion}

There are two possible ways for transferring the knowledge in the context of industry: one implies that expert operators directly teach apprentice ones, and another requires the use of any kind of interface to store the knowledge. The first approach has some drawbacks in a competitive environment such as industry: e.g., the need to gather ex pert and trainee operators in a place and time, and the potential use of production resources. Therefore, this work has been focused on the second approach, proposing a new immersive training system and comparing it with the most common system in the industries.

There are some differences between the proposed system and the conventional guides both in paper and digital formats. On the one hand, the developed system is able to collect the real behaviors of multiple operators, whereas the conventional ones usually consider the formal procedures for the assemblies. On the other hand, immersive devices enhance the perception of operators in comparison to books and screens, which may impact on the learning capacity of operators, as well as on their motivation in the training process.

Moreover, the experiments reported in previous sections shed some light on the matter of immersive training systems in the industry. The developed system provided the best results in terms of time and errors 


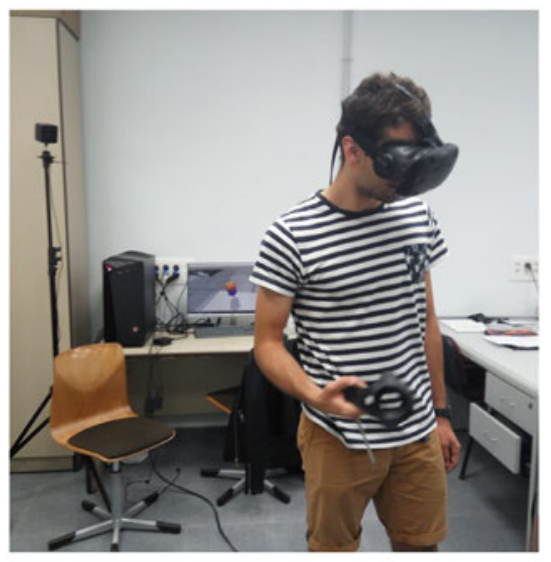

(a)

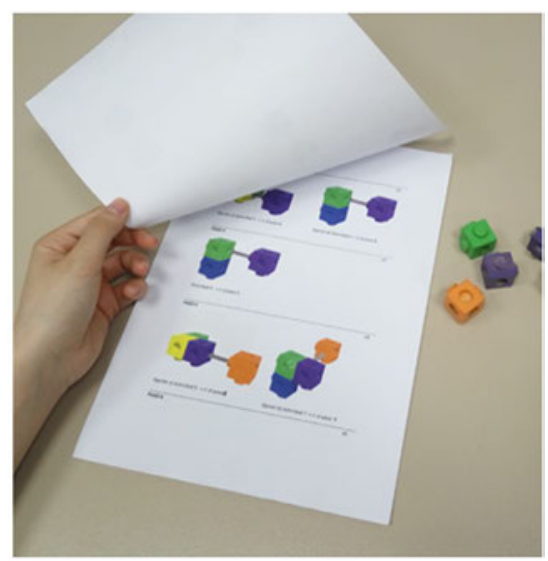

(b)

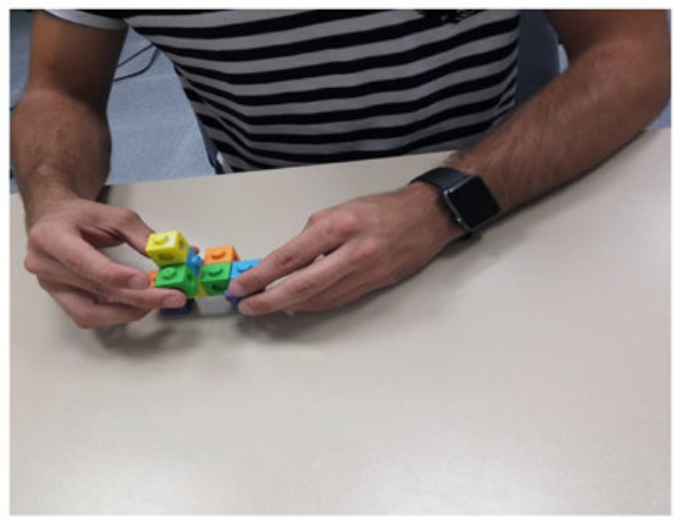

(c)

Fig. 10. Three pictures of the experiments: (a) immersive training system, (b) conventional guide and (c) final assembly.

Table 6

Performance scores according to guide and assembly.

\begin{tabular}{llllll}
\hline Guide & Assembly & Mean score & St. dev. & Norm. score & St. dev. \\
\hline Conventional & 1 & 57.8 & 39.7 & 0.1931 & 0.0693 \\
Immersive & 1 & 30.6 & 32.6 & 0.1162 & 0.0761 \\
Conventional & 2 & 65.8 & 40.5 & 0.2845 & 0.1601 \\
Immersive & 2 & 60.6 & 32.2 & 0.1995 & 0.0851 \\
Conventional & 3 & 58.9 & 44.1 & 0.1928 & 0.0757 \\
Immersive & 3 & 53.3 & 36.8 & 0.2185 & 0.1053 \\
Conventional & 4 & 96.7 & 67.0 & 0.3807 & 0.1366 \\
Immersive & 4 & 120.7 & 64.4 & 0.4145 & 0.1292 \\
\hline
\end{tabular}

in the simpler assemblies, whereas no significant differences were found in the more complex ones. The operators evaluated their ex perience with the immersive system better than with the conventional one, considering it significantly better in terms of mental demand (i.e., the mental and perceptual activity required to perform the task [40]), performance (i.e., the subjective impression about the performance in the task [40]), perception (i.e., the ability to perceive the different blocks and their places), learning (i.e., the subjective evaluation of learning during the process), and result (i.e., the subjective impression about the results of the test). All these results invite us to think opti mistically about the future of immersive training systems, although more experiments with more relevant scenarios are necessary to de termine their performance.

As previously mentioned, the proposed training system has been validated using a toy example with building blocks. Despite this fact, the training system has been developed taking into account future ap plications in the context of Industry 4.0. Therefore, the coding system,

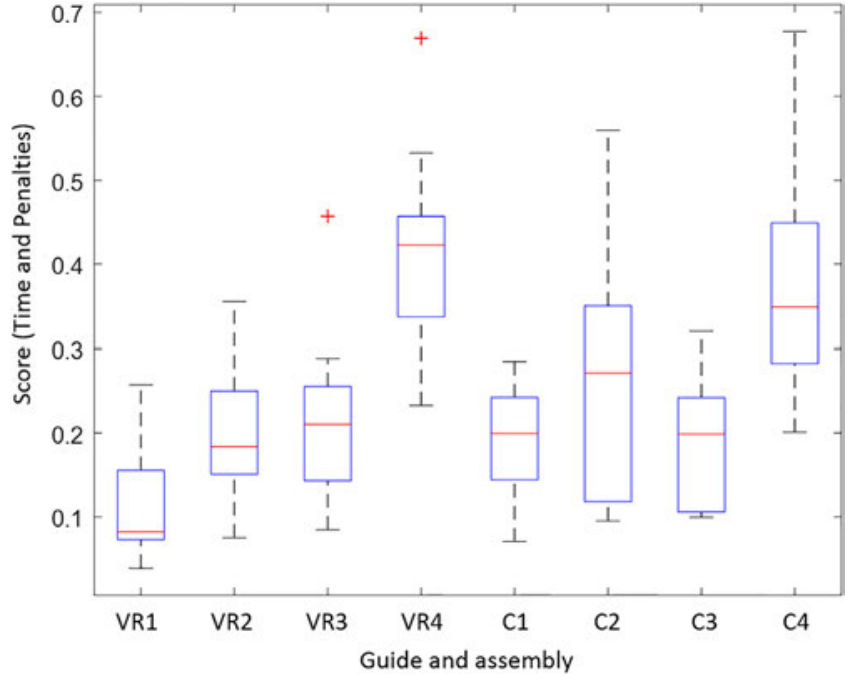

Fig. 11. Box and whisker plot for combinations of guide and assembly.

event, state, activity and task logs, transition systems, and expert and training interfaces are prepared for this potential use.

The coding system can be applied to blocks of different shapes and sizes without the need to make changes. However, if the blocks have more than six joints in total or more than one on the same side, the codes of joints must be changed, adding the required numbers and following the rules to reorder the chains. In addition, the equivalence between the joints and their numbers must be stored in the event logs to 
Table 7

Operator evaluations (from 1 "VR guide is much better" to 5 "Conventional guide is much better".

\begin{tabular}{lll}
\hline Variable & Mean evaluation & Std. dev. \\
\hline Mental demand & 2.15 & 1.23 \\
Physical demand & 3.55 & 1.43 \\
Temporal demand & 2.85 & 1.31 \\
Effort & 2.65 & 1.27 \\
Performance & 2.50 & 1.10 \\
Frustration & 2.90 & 1.02 \\
Perception & 1.40 & 0.50 \\
Learning & 1.95 & 1.23 \\
Result & 2.40 & 1.14 \\
\hline
\end{tabular}

be managed by the training interface.

Finally, if a tool is required to perform a step of the assembly, this tool can be added to the activity log, together with the active and target assemblies and the approach to join them. In this way, the operations are completely defined in the event logs and models, so the trainee operators have enough information to learn the assemblies.

A last interesting fact to consider is the cost to implement the pro posed system in an industry. The 3D models of the assemblies and their parts should be available, since they are needed for the design and production of them. Therefore, the effort should be focused on in troducing them in the immersive environment, as well as to adapt the coding system and event logs. The cost of VR headsets is decreasing as these devices are filling a gap in the entertainment industry and in creasing their potential consumers. Moreover, the longer scale of the industrial processes (taking into account the complexity of assembly and operators involved in them), the higher expected profitability of the proposed system. This is because the effort to implement the system increases slightly with the scale of the process, whereas the saving re lated to the participation of operators and the use of production re sources is significant.

\section{Conclusions}

This paper proposes a complete system for transferring the knowl edge from expert to apprentice operators in the context of Industry 4.0. The system provides an immersive interface based on virtual reality to both expert and apprentice operators. This interface faithfully re presents the real environment, improving the involvement of operators in the tasks and reducing the costs of working in a real environment (e.g., using the production line to teach or learn may imply temporally stop the production and put the equipment at risk). Additionally, techniques of process mining are used to collect and filter the data from experts, obtain models that represent the assemblies and teach new operators.

The developed system has been tested through a set of experiments in which twenty operators had to learn and build four assemblies. These tests were designed not only to estimate the performance of the pro posed system, but also to compare it to a conventional one. The results show that the performance of operators with VR guide is significantly better in one of the assemblies and cannot be considered better or worse in the other three. The questionnaires show that the operator evalua tions of VR guide are significantly better in terms of mental demand, performance, perception, learning, and result. These results confirm that the developed training system is competitive against the conven tional one, and the operators are receptive to this type of immersive system.

The future works will focus on applying the proposed system to more realistic assemblies. For this purpose, we would like to consider real cases, such as furniture, machines and robots. In this way, we will test the coding system with blocks of different shapes and sizes, checking if it works properly or changes are required. Additionally, more complex assemblies can lead to more varied experiments with more relevant results, since they require more time to be built, but, at the same time, can be easier to memorize. In this context, we will compare the performance of operators again using the proposed system and a conventional guide. Finally, a comparison between virtual reality and augmented reality in the context of industry should be interesting to determine the future of immersive training systems.

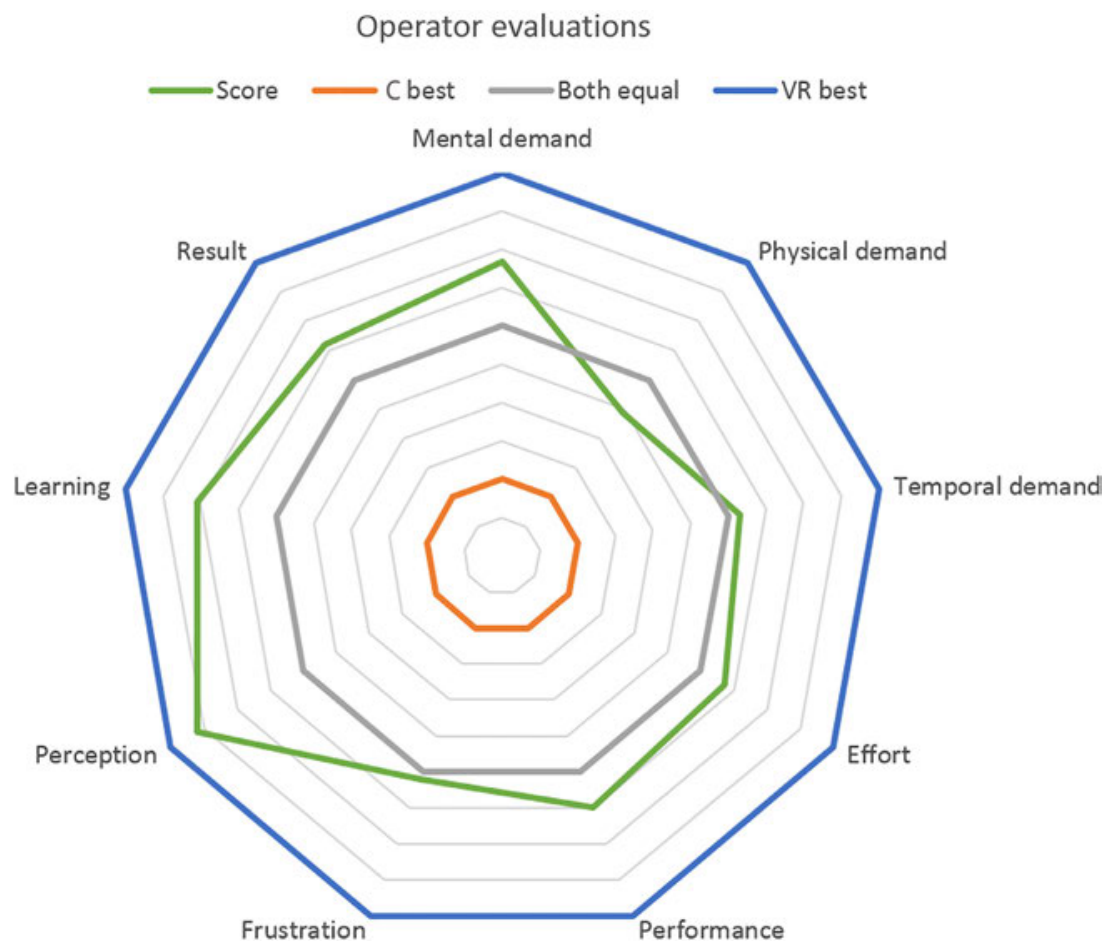

Fig. 12. Diagram with the evaluations of operators. 


\section{Acknowledgements}

The research leading to these results has received funding from RoboCity2030 III CM project (Robótica aplicada a la mejora de la ca lidad de vida de los ciudadanos. Fase III; S2013/MIT 2748), funded by Programas de Actividades I+D en la Comunidad de Madrid and co funded by Structural Funds of the EU; and from the DPI2014 56985 R project (Protección robotizada de infraestructuras críticas) funded by the Ministerio de Economía y Competitividad of Gobierno de España.

\section{Supplementary material}

Supplementary material associated with this article can be found, in the online version, at doi:10.1016/j.rcim.2019.05.004.

\section{References}

[1] M. Hermann, T. Pentek, B. Otto, Design principles for industrie 4.0 scenarios, System Sciences (HICSS), 2016 49th Hawaii International Conference on, IEEE, 2016, pp. 3928-3937.

[2] M. Rüßmann, M. Lorenz, P. Gerbert, M. Waldner, J. Justus, P. Engel, M. Harnisch, Industry 4.0: the future of productivity and growth in manufacturing industries, Boston Consult. Group 9 (2015).

[3] H. Inkinen, Review of empirical research on knowledge management practices and firm performance, J. Khnowl. Manag. 20 (2) (2016) 230-257.

[4] D. Hislop, R. Bosua, R. Helms, Knowledge Management in Organizations: a Critical Introduction, Oxford University Press, 2018.

[5] M. Bortolini, E. Ferrari, M. Gamberi, F. Pilati, M. Faccio, Assembly system design in the industry 4.0 era: a general framework, IFAC-PapersOnLine 50 (1) (2017) 5700-5705.

[6] J.J. Roldán, E. Peña-Tapia, D. Garzón-Ramos, J. de León, M. Garzón, J. del Cerro, A. Barrientos, Multi-robot systems, virtual reality and ros: developing a new generation of operator interfaces, Robot Operating System (ROS), Springer, 2019, pp 29-64.

[7] J.J. Roldán, E. Peña-Tapia, A. Martín-Barrio, M.A. Olivares-Méndez, J. Del Cerro, A. Barrientos, Multi-robot interfaces and operator situational awareness: study of the impact of immersion and prediction, Sensors 17 (8) (2017) 1720.

[8] K. Smparounis, D. Mavrikios, M. Pappas, V. Xanthakis, G.P. Viganò, K. Pentenrieder, A virtual and augmented reality approach to collaborative product design and demonstration, Technology Management Conference (ICE), 2008 IEEE International, IEEE, 2008, pp. 1-8.

[9] A.A. Kadir, X. Xu, E. Hämmerle, Virtual machine tools and virtual machiningatechnological review, Rob. Comput.-Integr. Manuf. 27 (3) (2011) 494-508.

[10] L. Rentzos, C. Vourtsis, D. Mavrikios, G. Chryssolouris, Using vr for complex product design, International Conference on Virtual, Augmented and Mixed Reality, Springer, 2014, pp. 455-464.

[11] N.E. Seymour, A.G. Gallagher, S.A. Roman, M.K. O’Brien, V.K. Bansal, D.K. Andersen, R.M. Satava, Virtual reality training improves operating room performance: results of a randomized, double-blinded study, Ann. Surg. 236 (4) (2002) 458

[12] P. Piromchai, A. Avery, M. Laopaiboon, G. Kennedy, S. O'Leary, Virtual reality training for improving the skills needed for performing surgery of the ear, nose or throat., 2015.

[13] T. Gunn, P. Rowntree, L. Nissen, P. Bridge, The impact of virtual reality training on medical imaging students' confidence and general radiographic skills, JMRS 63 (S1) (2016) 28.

[14] K. Alexopoulos, D. Mavrikios, G. Chryssolouris, Ergotoolkit: an ergonomic analysis tool in a virtual manufacturing environment, Int. J. Comput. Integr. Manuf. 26 (5) (2013) 440-452.

[15] G. Chryssolouris, D. Mavrikios, D. Fragos, V. Karabatsou, A virtual reality-based experimentation environment for the verification of human-related factors in assembly processes, Rob. Comput.-Integr. Manuf. 16 (4) (2000) 267-276.

[16] F. Pallavicini, L. Argenton, N. Toniazzi, L. Aceti, F. Mantovani, Virtual reality applications for stress management training in the military, Aerosp. Med. Hum. Perform. 87 (12) (2016) 1021-1030.

[17] K.K. Bhagat, W.-K. Liou, C.-Y. Chang, A cost-effective interactive 3d virtual reality system applied to military live firing training, Virtual Real. 20 (2) (2016) 127-140.

[18] E.D. Ragan, D.A. Bowman, R. Kopper, C. Stinson, S. Scerbo, R.P. McMahan, Effects of field of view and visual complexity on virtual reality training effectiveness for a visual scanning task, IEEE Trans. Visual. Comput. Graph. 21 (7) (2015) 794-807.

[19] M.J. Smith, L. Boteler Humm, M.F. Fleming, N. Jordan, M.A. Wright, E.J. Ginger, K. Wright, D. Olsen, M.D. Bell, Virtual reality job interview training for veterans with posttraumatic stress disorder, J. Vocational Rehabilit. 42 (3) (2015) 271-279.

[20] N. Gavish, T. Gutiérrez, S. Webel, J. Rodríguez, M. Peveri, U. Bockholt, F. Tecchia, Evaluating virtual reality and augmented reality training for industrial maintenance and assembly tasks, Interact. Learn. Environ. 23 (6) (2015) 778-798.

[21] S. Pedram, P. Perez, S. Palmisano, M. Farrelly, A systematic approach to evaluate the role of virtual reality as a safety training tool in the context of the mining industry(2016).

[22] A. Grabowski, J. Jankowski, Virtual reality-based pilot training for underground coal miners, Safety Sci. 72 (2015) 310-314.

[23] H. Fang, S. Ong, A. Nee, Interactive robot trajectory planning and simulation using augmented reality, Rob. Comput.-Integr. Manuf. 28 (2) (2012) 227-237.

[24] J. Cecil, D. Powell, D. Vasquez, Assembly and manipulation of micro devices - a state of the art survey, Rob. Comput.-Integr. Manuf. 23 (5) (2007) 580-588.

[25] H. Zhang, Head-mounted display-based intuitive virtual reality training system for the mining industry, Int. J. Mining Sci. Technol. 27 (4) (2017) 717-722.

[26] D. Mavrikios, V. Karabatsou, D. Fragos, G. Chryssolouris, A prototype virtual reality-based demonstrator for immersive and interactive simulation of welding processes, Int. J. Comput. Integr. Manuf. 19 (03) (2006) 294-300.

[27] G. Chryssolouris, D. Mavrikios, D. Fragos, V. Karabatsou, K. Pistiolis, A novel virtual experimentation approach to planning and training for manufacturing processes-the virtual machine shop, Int. J. Comput. Integr. Manuf. 15 (3) (2002) 214-221.

[28] W.M. Van der Aalst, Process Mining: Data Science in Action, Springer, 2016.

[29] W.M. van der Aalst, V. Rubin, B.F. van Dongen, E. Kindler, C.W. Günther, Process mining: a two-step approach using transition systems and regions, BPM Center Rep. BPM-06-30, BPMcenter. org 6 (2006).

[30] W.M. Van der Aalst, The application of petri nets to workflow management, J. CircuitsSyst. Comput. 8 (01) (1998) 21-66.

[31] R.M. Dijkman, M. Dumas, C. Ouyang, Semantics and analysis of business process models in bpmn, Inf. Software Technol. 50 (12) (2008) 1281-1294.

[32] W. Van Der Aalst, A. Adriansyah, B. Van Dongen, Causal nets: a modeling language tailored towards process discovery, International conference on concurrency theory, Springer, 2011, pp. 28-42.

[33] R.S. Mans, M. Schonenberg, M. Song, W.M. van der Aalst, P.J. Bakker, Application of process mining in healthcare-a case study in a dutch hospital, International join conference on biomedical engineering systems and technologies, Springer, 2008, pp. 425-438.

[34] W.M. van der Aalst, H.A. Reijers, A.J. Weijters, B.F. van Dongen, A.A. De Medeiros, M. Song, H. Verbeek, Business process mining: an industrial application, Inf. Syst. 32 (5) (2007) 713-732.

[35] A. Rozinat, I.S. de Jong, C. Gunther, W.M. van der Aalst, Process mining applied to the test process of wafer scanners in asml, IEEE Trans. Syst. Man Cybern. Part C (Appl. Rev.) 39 (4) (2009) 474-479.

[36] J.J. Roldán, M.A. Olivares-Méndez, J. del Cerro, A. Barrientos, Analyzing and improving multi-robot missions by using process mining, Autonom. Robots 42 (6) (2018) 1187-1205.

[37] J.J. Roldán, J. del Cerro, A. Barrientos, Using process mining to model multi-uav missions through the experience, IEEE Intell. Syst. 32 (4) (2017) 40-47.

[38] S.G. Hart, Nasa-task load index (nasa-tlx); 20 years later, Proceedings of the human factors and ergonomics society annual meeting, 50 Sage Publications Sage CA: Los Angeles, CA, 2006, pp. 904-908.

[39] H.P. Martinez, G.N. Yannakakis, J. Hallam, Don't classify ratings of affect; rank them!, IEEE Trans. Affective Comput. 5 (3) (2014) 314-326.

[40] D. Schuff, K. Corral, O. Turetken, Comparing the understandability of alternative data warehouse schemas: an empirical study, Decis. Supp. Syst. 52 (1) (2011) 9-20. 